\title{
THE SOMETIMES DELETERIOUS INFLUENCE OF THE BRACHIO- RADIALIS RADIAL EXTENSORS AND FINGER FLEXORS ON RESIDUAL HAND FUNCTION IN SPASTIC TETRAPLEGICS
}

\author{
By Walter J. Treanor, M.D. and Lloyd W. Taylor, M.D. \\ St. Mary's Hospital and Medical Center, San Francisco, California, U.S.A.
}

\section{THE BRACHIORADIALIS AND RADIAL WRIST EXTENSORS}

OVER a 20-year period, some interesting clinical observations have been made on the actions of these muscles in a series of 42 quadriparetic patients, with complete sparing of the C6, and complete or partial sparing of the $\mathrm{C}_{7}$, myotomes. The presence of an intact or 'unbraked' brachioradialis frequently interfered with proper use of residual wrist dorsiflexor function. It was noted that most of these patients initiated prior flexion at the elbow, usually in the semi-prone position, which compromised residual function of the dorsiflexor 'hinge hand' in picking up of small and medium size objects.

In the first operated patient, a retained (and unbraked) action of the radial wrist extensors interfered with adequate spread of the thumb and index. Prior to operation, this patient was barely able to dorsiflex the wrist through a $10^{\circ}$ range. Because of this inadequate range, it was decided that transposition of the brachioradialis into the extensor carpi ulnaris might conceivably improve the excursion of dorsiflexion, and reduce the tendency towards radial deviation. This procedure was done under local anaesthetic. Post-operatively, there was an appreciable reduction of the previous radial deviation at the wrist, an increase of dorsiflexion range from $10^{\circ}$ to $40^{\circ}$, and reduction in speed of prior elbow flexion. Follow-up pictures of the patient, taken four months and I4 years later, clearly showed improvement in the alignment of the wrist and in function of the wrist dorsiflexor hinge action. The feared problem of persistent flexion contracture at the elbow has been overcome by continued passive stretching throughout this period.

In the C8-preserved hand, the reflex overactivity of the brachioradialis muscle, which was shown electromyographically to be electrically 'silent' only in the midprone position, significantly impaired the patient's capacity to flex and extend his fingers. Clonus would rapidly develop once the brachioradialis was activated even by minor movements of pronation or supination. This over-responsiveness occurred maximally in the finger and thumb flexors. It reduced the patient's capacity to pick up a glass, or to bring objects smoothly to his mouth. Excision of a 7 inch segment to the brachioradialis muscle and tendon was performed. The consequent improvement in the use of the finger flexors was well demonstrated in the motion pictures following surgery.

As a consequence of this serendipitous discovery, we have used this method of wide excision, or transposition of the brachioradialis muscle into the flexor carpi ulnaris, in a large number of patients, both to reduce the tendency toward supination of the forearm, and to damp down finger flexor overactivity. This transposition 
technique has also been employed in hemiplegic patients, to provide a pronation alignment of the forearm, by reducing the combined supination drive of the biceps and brachioradialis. The relatively useless supinated 'receiving hand' has thus been converted into a more functional pronate 'giving hand' (Daniel, personal communication). A rotation osteotomy of the forearm has been employed in two patients for the same purpose.

\section{THE FINGER FLEXORS}

A deleterious effect of reflexly overactive finger flexors on wrist dorsiflexor action has been noted in patients, in whom there is a rapid transition from hypofunctioning or normally functioning muscles to hyperreflexic muscles. There may be a rapid transition from muscle hypofunction to reflex hyperactivity of the finger flexors within one segment, particularly when the $\mathrm{C}_{7}$ and $\mathrm{C} 8$ roots are partially spared. It becomes difficult to splint these patients adequately because of this flexor hyperreflexia. In these patients we chose to elongate the finger and thumb flexors, as a primary procedure, in order to permit a more physiological posture of the phalanges. By reducing the antagonistic action of finger flexor overactivity, there was a noticeable augmentation of the excursion and power of the wrist dorsiflexors. Pre-operative evaluation of the expected surgical improvement could be assessed more critically by prior local anaesthetic blocking of the median and ulnar nerves around the elbow. These blocks are helpful in disclosing the true residual capabilities of the wrist dorsiflexors, when unopposed by hyperactive finger flexors. Surgical elongation of the finger and thumb flexors at the wrist is proposed as a substitute for the more commonly employed mechanical means of augmenting weak wrist dorsiflexors by use of electric or spring assistive bracing. It is also suggested, in prophylactic use, to prevent the dread complication of flexor contracture of the fingers, which has been seen in many patients who could otherwise have had a useful 'flexor hinge hand'.

\section{CONCLUSIONS}

I. Too little attention has been paid to the influence of the brachioradialis muscle's effect on retained wrist function in certain types of quadriplegia. Although this muscle has no anatomical function in the use of the hand, it can reflexly alter the effectiveness of retained wrist dorsiflexion and finger extension. The phenomenon of 'inversion' of the brachioradialis reflex into the finger flexors has been known by diagnosticians for many years (Wartenberg, I945). It is postulated that this 'inversion' is also present during active torque movements of the radioulnar joints (Treanor \& Reifenstein, 196I). Support of this hypothesis of the brachioradialis as a 'remote source of hyperreflexia' has been obtained by electrophysiological methods.

2. As a consequence, we feel satisfied that there is good justification for

(a) Transposition of the brachioradialis to augment a weakened or absent ulnar wrist extensor.

(b) Its translocation to the flexor carpi ulnaris to reduce an excessively supinated kinetic posture of the forearm, and to provide the more functional posture of pronation. 
(c) Release of a large segment of the muscle and tendon when rotation movements of the forearm, by 'inversion', cause reflex over-activity of the finger and thumb flexors.

(d) Elongation of the finger and thumb flexors, when their reflex over-activity interferes with the action of wrist dorsiflexion.

\section{REFERENCES}

Daniel, M. V. Personal communication.

TREANOR, WALTER J. (I963). The role of physical medicine treatment in stroke rehabilitation. Clin. Ortho. 63, 14-22.

WARTENBERG, R. (1945). The examination of reflexes: a simplification. Year Book Publishers, Chicago.

Treanor, W. J. \& ReifensteIn, G. (I96I). Potential reversibility of the hemiplegic posture. Amer. F. Cardiology, 7, 370-378. 